\title{
Dengue Fever: \\ Two Unexpected Findings
}

Matthew A. Heimann, MD; Browning S. Wayman, MD; Ross M. Vander Noot, MD; Scott W. Irvine, MD, MPH

\section{These two case reports highlight the importance of maintaining a high index of suspicion for dengue in febrile patients with a history of recent travel to countries where this disease is endemic.}

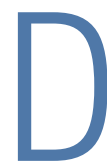

engue fever is the most commonly transmitted arboviral disease in the world, affecting an estimated 2.5 billion people who live in areas endemic to the virus. This exposure yields an annual incidence of 100 million cases of dengue, which translates into 250,000 cases of hemorrhagic fever. With an expanding geographic distribution and increasing number of epidemics, the World Health Organization (WHO) has classified dengue as a major public health concern. ${ }^{1}$ Enhanced globalization and changing climate patterns have resulted in a dramatic increase in the incidence of dengue in both North and Central America. Aggregate North and Central American data from 2010 to the present revealed over 1.7 million cases of dengue, nearly 80,000 of which were severe, and 747 deaths. ${ }^{2}$ Based on these statistics, dengue fever should be considered in the differential diagnosis of febrile ED patients in the developed world who had a history of recent travel. We present two cases that highlight the com- plexity of diagnosis and novel complications associated with dengue fever.

\section{Case Reports}

\section{Case 1}

A 24-year-old man presented to the ED with a 4-day history of intermittent fever of up to $102.02^{\circ} \mathrm{F}$, which was accompanied by chills, myalgia, and rigors. The patient stated that he had visited Vietnam, Thailand, Indonesia, and Malaysia 8 days prior to presentation, and had experienced mosquito bites daily throughout his travels. $\mathrm{He}$ further noted that his symptoms had improved on day 3 of his illness, but acutely worsened on day 4 , which prompted him to visit the ED. The patient's primary complaint was a severe retro-orbital headache, fever, and one episode of epistaxis.

On physical examination, the patient had conjunctivitis and hepatosplenomegaly, but otherwise appeared well. His laboratory evaluation was significant for leukopenia (white blood cell [WBC] count, $2.40 \times 10^{\%} / \mathrm{L}$ ), thrombocytopenia (platelet

Dr Heimann is a resident, department of emergency medicine, University of Alabama at Birmingham. Dr Wayman is an international emergency medicine fellow, department of emergency medicine, University of Alabama at Birmingham. Dr Vander Noot is an associate professor, department of emergency medicine; codirector, division of global health; and codirector, international emergency medicine fellowship, University of Alabama at Birmingham. Dr Irvine is an assistant professor, department of emergency medicine; codirector, division of global health; and codirector, international emergency medicine fellowship, University of Alabama at Birmingham. 
count, $123 \times 10^{9} / \mathrm{L}$ ), and a positive mononuclear spot test. Both dengue immunoglobulin $\mathrm{G}$ ( $\mathrm{IgG}$ ) and immunoglobulin $\mathrm{M}$ (IgM) tests sent from the ED were negative. Based on the patient's thrombocytopenia and epistaxis, as well as concerns that the patient was entering into the critical phase of dengue fever, he was admitted to the inpatient hospital for observation.

The patient's course improved during his stay with symptomatic treatment and bloodcount monitoring, and he was discharged home on hospital day 3. He followed up at our hospital travel clinic the day after discharge; a repeat dengue IgM test taken during this visit came back positive.

\section{Case 2}

A 51-year-old man presented to the ED with a 3-day history of intermittent fever and diffuse myalgia. He reported chills, night sweats, and the feeling of abdominal fullness. He denied nausea, vomiting, or changes in the character of his stool. He had no known sick contacts, but reported he had traveled from the Philippines 3 days prior to presentation and that his symptoms had developed en route to the United States. The patient also denied any known tick, mosquito, or animal exposures. He said he had treated his symptoms with acetaminophen and nonsteroidal anti-inflammatory drugs. Prior to his arrival at the ED, he had twice presented to a walk-in clinic earlier that day. Repeated laboratory testing at the ED showed a decrease in WBC count from $42.0 \times 10^{9} / \mathrm{L}$ to $31.0 \times 10^{9} / \mathrm{L}$, as well as a declining platelet count from $123 \times 10^{9} / \mathrm{L}$ to $87 \times 10^{9} / \mathrm{L}$. On physical examination, the patient was ill-appearing, diaphoretic, and had a temperature of $100.6^{\circ} \mathrm{F}$. His vital signs were otherwise within normal limits.

With the exception of a mild diffuse petechial rash on the patient's thighs bilaterally, the physical examination was unrevealing. A tourniquet test (TT) to assess capillary fragility was performed at bedside, and yielded a positive result

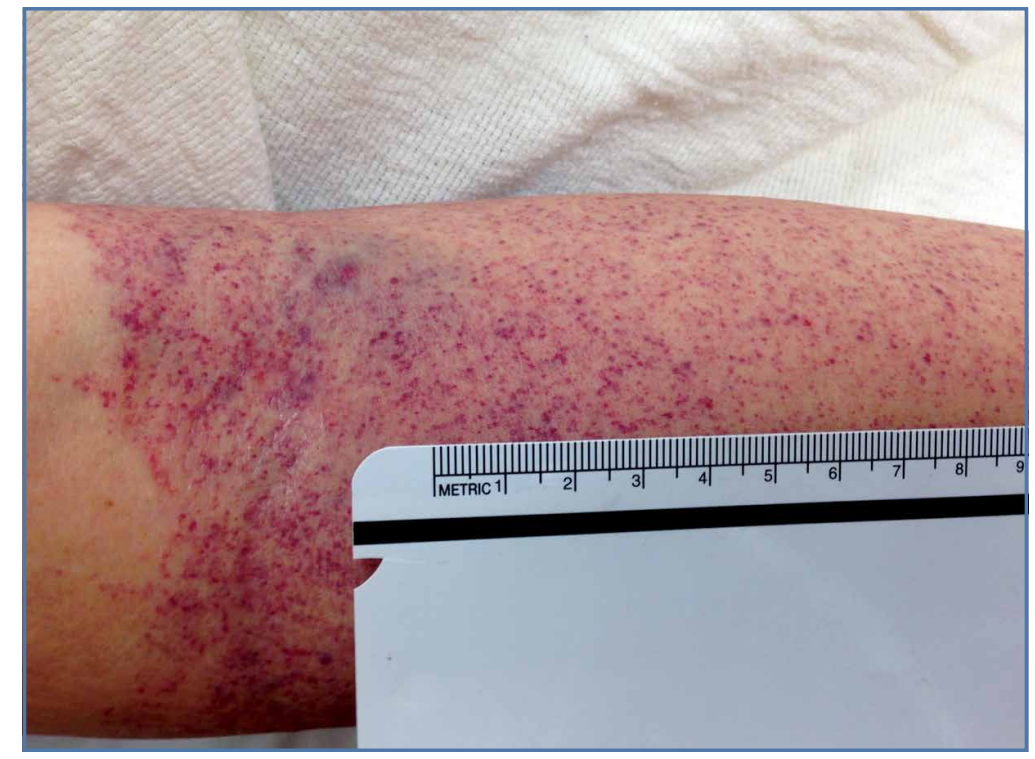

Figure 1. Positive tourniquet test result of the volar forearm showing greater than 10 petechiae per 1 -inch $(2.5-\mathrm{cm})$ square below the antecubital fossa.

(Figure 1). Work-up further demonstrated a declining WBC of $2.70 \times 10^{9} / \mathrm{L}$ and declining platelet count of $65 \times 10^{9} / \mathrm{L}$.

A polymerase chain reaction (PCR) test confirmed a diagnosis of dengue, with a positive dengue type-4 (DEN-4) serotype detection. Supportive care was initiated, and the patient was admitted to the inpatient hospital for continued treatment. $\mathrm{He}$ was discharged home on hospital day 5 ; however, he returned to the ED later that day with increasing headache and left flank pain. Work-up included axial and coronal computed tomography scans of the abdomen and pelvis, which revealed hematuria and a left upper pole renal infarction surrounded by mild perinephric fat stranding (Figure 2a and 2b) with maintenance of left renal artery/vein patency.

The patient was admitted to an inpatient floor, where symptomatic management was employed. He underwent unrevealing bubble echocardiography and lower extremity Doppler ultrasound imaging, and anticoagulation therapy was initiated per a consultation with hematology services. The patient was discharged home in improved, stable condition on hospital day 8 . 

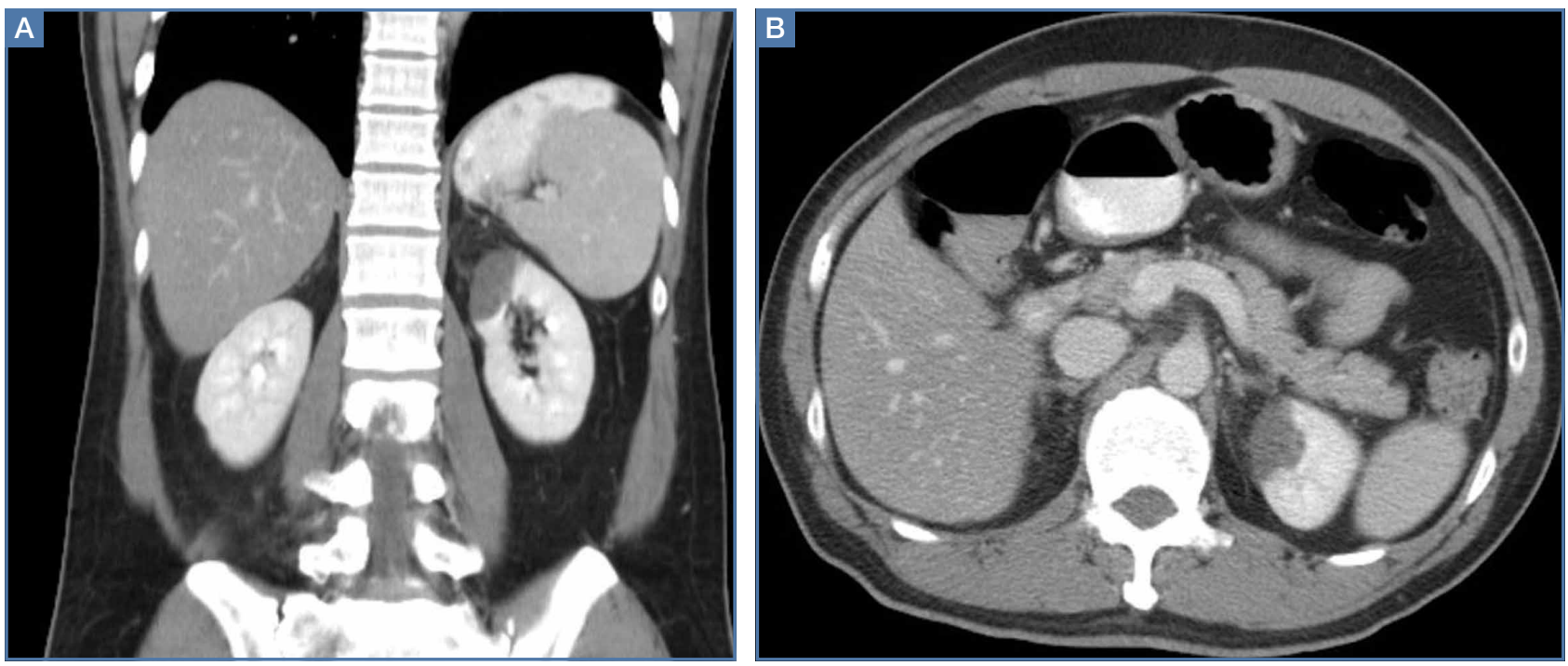

Figure 2. (A) Left renal coronal computed tomography (CT) scan and (B) axial CT scan of patient in case report 2, demonstrating left upper pole renal Infarct.

\section{Discussion}

Dengue virus is a single-stranded, nonsegmented RNA virus in the Flaviviridae family. Four major subtypes exist: DEN1, DEN-2, DEN-3, and DEN-4. Lifelong serotype-specific immunity is conferred following infection. The virus is transmitted by the female Aedes aegypti mosquito, which is found worldwide but has a predilection for tropical and subtropical regions. The Aedes aegypti mosquito remains an effective vector secondary to its diurnal feeding habit and nearly imperceptible bite..$^{1,3}$

The viral incubation period for dengue is typically 3 to 7 days ${ }^{4}$; therefore, dengue is highly unlikely in patients whose symptoms begin more than 2 weeks after departure from an endemic area. Replication primarily occurs in the regional lymph nodes and disseminates through the lymphatic system and bloodstream. ${ }^{1}$

The 1997 WHO guidelines previously classified dengue into three categories: undifferentiated fever, dengue fever, and dengue hemorrhagic fever (which was further classified by four severity grades, with grades III and IV defined as dengue shock syndrome). However, changes in epidemi- ology of the disease and reports of difficulty applying the criteria in the clinical setting led to reclassification of dengue on a continuum from dengue to severe dengue in the WHO's updated 2009 guidelines. ${ }^{4}$

\section{Signs and Symptoms}

The ramifications of dengue infection can range from asymptomatic (typically in young, immunocompetent patients) to lethal. Key symptoms of dengue fever include nausea, vomiting, fever, respiratory symptoms, morbilliform or maculopapular rash, and headache or retro-orbital pain. In addition, arthralgia (hence the colloquial name for dengue of "breakbone fever"), myalgia, and conjunctivitis may exist. ${ }^{3,4}$ Fever usually lasts 5 to 7 days and can be biphasic, with a return of symptoms after the initial resolution as seen in case report $1 .{ }^{4}$ Severe dengue is characterized by capillary leakage, hemorrhage, or end-organ damage. ${ }^{3-5}$ The most common bleeding sites are the skin, nose, and gums.

\section{Diagnosis}

Bedside evaluation for dengue can be performed with the TT-one of the WHO's case definitions for dengue. ${ }^{6}$ This is accom- 
plished by placing a manual blood pressure (BP) cuff on the arm and inflating it to halfway between systolic and diastolic BP for 5 minutes. The test is positive for dengue if more than 10 petechiae appear per 1-inch $(2.5-\mathrm{cm})$ square below the antecubital fossa. ${ }^{7}$ Of note, the test has poor sensitivity $(51.6 \%, 95 \%$ confidence interval [CI], 33-69), but good specificity (82.4\%, 95\% CI, 76-87). ${ }^{7,8}$ A positive TT combined with leukopenia increases the sensitivity to 93.9\%, [95\% CI, 89-96]. ${ }^{7}$ While not specific to dengue infection, in the right clinical scenario, the TT is a simple bedside test to help confirm the diagnosis and is extremely useful in resource-limited settings.

During the initial days of illness, the virus may be detected by PCR, as viremia and fever usually correlate. Once defervescence occurs, IgM and then IgG antibodies become detectable. When using these antibody tests to evaluate for dengue, clinicians should be aware of cross-reactivity with other flavivirus infections, such as yellow fever or Japanese encephalitis (including immunological cross-reactivity). ${ }^{1}$ New diagnostic modalities include enzyme immunoassays that can detect dengue viral RNA within 24 to 48 hours, and viral antigen-detection kits, which can yield results in less than 1 hour. ${ }^{4}$

Aside from advanced laboratory testing, worsening thrombocytopenia in light of a rising hematocrit can be highly suggestive of dengue. Leukopenia with lymphopenia and mild elevation of hepatic enzymes (typically 2 to 5 times the upper limits of the normal reference range) are also often seen in active infections. ${ }^{1}$ The occurrence of these signs in conjunction with a rapid reduction in the platelets often signals transition to the critical phase of plasma leakage. ${ }^{1,4}$

\section{Treatment}

Treatment of dengue consists of supportive care and transfusion when necessary. The WHO recommends strict observation of patients with suspected dengue who have warning signs of severe disease (eg, abdominal pain, persistent vomiting, muco- sal bleeding, lethargy, hepatomegaly, rapid increase in hematocrit with concomitant drop in platelet count). Inpatient treatment centers on judicious fluid management, trending blood count parameters, and monitoring for signs of plasma leakage and hemorrhage. Fluid resuscitation is titrated to optimize central and peripheral circulation and end-organ perfusion. Blood-product administration should be reserved for suspected or severe bleeding. ${ }^{4}$

While dengue fever was the final diagnosis in both of our case presentations, these cases also highlight key diagnostic and treatment dilemmas associated with dengue. The patient in the first case report demonstrated the characteristic biphasic fever seen with dengue-resolution of symptoms on day 3 , but then return of fever and symptoms on day 4 . Often the dengue-specific antibodies are not formed until after the resolution of fever. This patient represents a classic example of dengue as the serologic studies sent on day 4 of the patient's illness were negative but then turned positive on day 7 , illustrating the need for high clinical suspicion and underscoring the importance of initiating treatment despite laboratory confirmation.

Further, regarding the patient in the second case, though proteinuria, hematuria, acute renal failure, and glomerulonephritis are previously described renal complications of dengue, ${ }^{9}$ a thorough literature search yielded no prior published accounts of renal infarction. Given the patient's previous healthy status and the lack of other hypothesis as to the mechanism of injury, we suspect this patient's renal infarction was due to the transient hypercoagulability characteristic of dengue and responsible for other clinical manifestations of the disease.

\section{Conclusion}

In addition to more prevalent illnesses such as malaria, acute traveler's diarrhea, and respiratory tract infections, dengue fever should be included in the differential diagnosis when evaluating a febrile patient 
who has a history of recent travel to countries where dengue is endemic. A high clinical suspicion, combined with a thorough history and physical examination, is essential to making the diagnosis.

Both of our case reports demonstrate some of the diagnostic limitations in the acute setting, and the breadth of clinical complications that can occur in this complex disease. With the increasing prevalence of dengue fever in North and Central America, it is likely that patients with the disease will present to EDs in the United States. Early diagnosis and awareness of potential complications can lead to timely initiation of life-saving supportive care.

\section{References}

1. Wilder-Smith A, Schwartz E. Dengue in travelers. N Engl J Med. 2005;353(9):924-932. dpo:10.1056/ NEJMra041927

2. Pan American Health Organization, World Health Organization. Number of reported cases of dengue and severe dengue (SD), Region of the Americas (by country and subregion). Washington, DC: Pan American Health Organization. http://www.paho. org/hq/index.php?option=com_docman\&task=doc view\&Itemid=\&gid=35610\&lang=es. Updated August 5, 2016. Accessed August 17, 2016.

3. Whitehorn J, Farrar J. Dengue. Clin Med (Lond). 2011;11(5):483-487

4. World Health Organization. Dengue: Guidelines for Diagnosis, Treatment, Prevention and Control. New Edition. Geneva, Switzerland: World Health Organization; 2009. http://www.who.int/tdr/publications/documents/ dengue-diagnosis.pdf. Accessed August 17, 2016.

5. Halstead SB. Dengue. Lancet. 2007;370(9599):16441652. doi:10.1016/S0140-6736(07)61687-0.

6. Centers for Disease Control and Prevention. Dengue Clinical Case Management E-learning. http://www. cdc.gov/dengue/training/cme/ccm/page73112.html; http://www.cdc.gov/dengue/training/cme/ccm/Tourniquet\%20Test_F.pdf. Accessed August 17, 2016.

7. Gregory CJ, Lorenzi OD, Colón L, et al. Utility of the tourniquet test and the white blood cell count to differentiate dengue among acute febrile illnesses in the emergency room. PLoS Negl Trop Dis. 2011;5(12):e1400.

8. Mayxay M, Phetsouvanh R, Moore CE, et al. Predictive diagnostic value of the tourniquet test for the diagnosis of dengue infection in adults. Trop Med Int Health. 2011;16(1):127-133.

9. Lizarraga KJ, Nayer A. Dengue-associated kidney disease. J Nephropathol. 2014;3(2):57-62. 